\title{
Yaşlanma Karşıtı Kozmetikler İçin Taşıyıcı Sistemler Olarak Yeni Yumuşak Malzemelerin Kullanımı
}

\section{Use of new soft material as a carrier system for anti-aging Cosmetics}

\author{
Mehmet Çolak ${ }^{1}$ \\ ${ }^{1}$ Dicle Üniversitesi, Fen Fakültesi Kimya Bölümü, Diyarbakır, mcolak@dicle.edu.tr
}

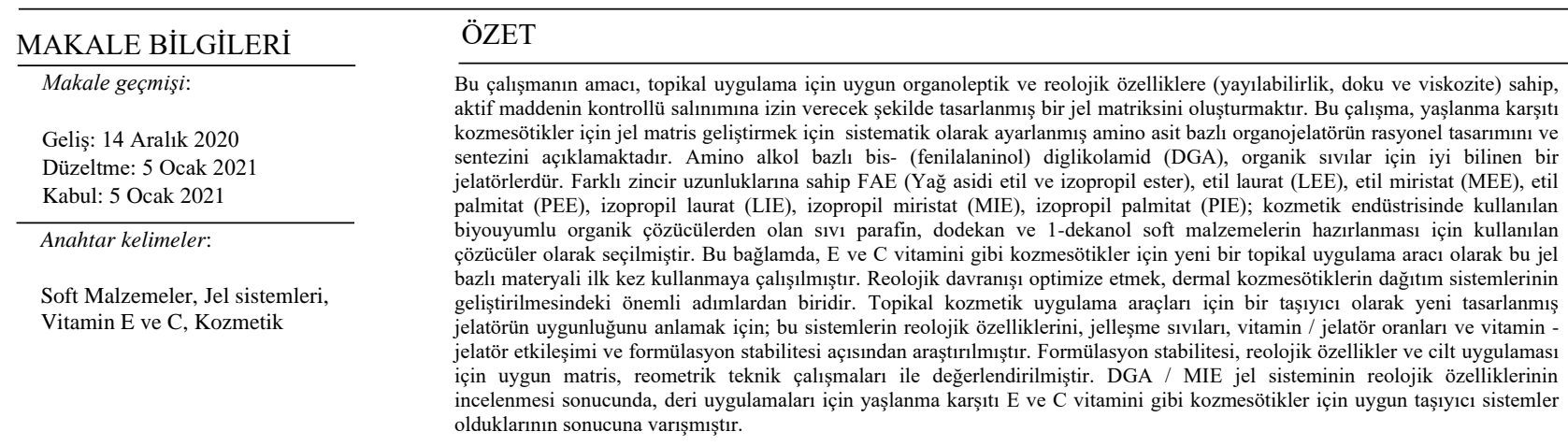

Doi: $10.24012 /$ dumf. 840481

\begin{tabular}{|c|c|}
\hline ARTICLE INFO & ABSTRACT \\
\hline Article history: & \multirow{6}{*}{$\begin{array}{l}\text { The aim of this work was to identify a gel with suitable organoleptic and rheological properties (spreadability, texture and viscosity) for } \\
\text { topical administration, designed to allow controlable release of the active principle. In this study describes the rational design and synthesi } \\
\text { of amino acid-based organogelator, which were systemically fine-tuned at the head group to develop gel matrix for anti-aging cosmeceutical. } \\
\text { A great variety of organic self-assemblies are known and several of them provide cosmeceutical delivery matrices. Amino alcohol based bis- } \\
\text { (phenylalaninol)diglycoholamide (DGA), a well-known LMWGs for organic fluids whose properties have been reported in several papers, } \\
\text { has been explored to develop depot systems and illustrated as a topically delivery vehicle for well known anti aging vitamine E and vitamine } \\
\text { C. FAE (Faty acid ethyl and isopropyl ester) with different chain lenghts, ethyl laurate (LEE), ethyl myristate (MEE), ethyl palmitate (PEE), } \\
\text { isopropyl laurate (LIE), isopropyl myristate (MIE), isopropyl palmitate (PIE); liquid paraffine, dodecane and 1-decanol were chosen as } \\
\text { biocompatible organic fluids which are used in cosmetic industry. In this context, we attempted for the first time, using these gel base } \\
\text { material as a novel topical delivery vehicle for cosmeceuticals. Optimizing rheological behavior is therefore one of the crucial steps in } \\
\text { development of dermal cosmeceuticals delivery systems. In order to understand the convenience of newly designed gelator as a carrier for } \\
\text { topical cosmeceutical delivery vehicles; we investigated rheological properties of these systems with respect to the, the gelation fluids, } \\
\text { vitamine/ gelator ratios and vitamine - gelator interaction and formulation stability. Formulation stability, rheological properties and matrix } \\
\text { convinent for skin application were evaluated with IR spectroscpy reometric techniqe Fourier transform infrared spectrophotometric studies } \\
\text { indicated absence of gelator-drug chemical interactions. The rheological properties of DGA/ MIE gel system was implied as a carrier anti } \\
\text { aging cosmeceutical for skin application. }\end{array}$} \\
\hline Received: 14 December 2020 & \\
\hline Revised: 5 January 2021 & \\
\hline Accepted: 5 January 2021 & \\
\hline Keywords: & \\
\hline $\begin{array}{l}\text { Bridge pier, local scour, clear } \\
\text { water scour, steady flow }\end{array}$ & \\
\hline
\end{tabular}

* Sorumlu yazar / Correspondence

Mehmet Çolak

$\bowtie$ mcolak@dicle.edu.tr 


\section{Giriș}

Kozmesötik terimi, kozmetik veya ilaç olarak kabul edilemeyen aktif maddeler içeren ürünleri tanımlamak için 25 y1ldan uzun bir süre önce oluşturulmuştur. Bunlardan biri olan E Vitamini ( $\alpha$-tokoferol) lipitte çözünür ve ağızdan alındığında membran lipidlerini peroksidasyona karş1 korur. Deride, UV'ye maruz kaldıktan sonra güneș yanığ 1 hücrelerini azalttığ 1 , serbest radikalleri nötralize ettiği ve ayrıca nemlendirici görevi gördüğü gösterilmiştir. Topikal kullanım amaciyla $\mathrm{C}$ ve E Vitaminlerinin birleştirilmesinin, kendi antioksidanlarının yanı sıra foto koruyucu etkilerini de arttırdığına dair önemli kanıtlar vardır [1]. Topikal olarak uygulanan vitaminleri içeren kozmetikler cilt bakımında artan bir role sahiptir. Kozmesötik formülasyonların birçoğu $C$ vitamini ve / veya $E$ vitamini içerse de, çok azı aslında cilt uygulamasında etkilidir. Bu bağlamda yeni soft malzeme olan Dioksalamidin (DOA) analogu olan Bis-digycolamide (DGA) bu çalışmada kozmesötik bir taşıyıcı sistem olarak değerlendirilmiştir. Aynı zaman bu çalışma, geleneksel organojellerin geliştirilmesi için alternatif olarak Yăg asidi etil ve izopropil esterler içeren DGA jellerini sunmaktadır. Farklı zincir uzunluklarına sahip Yağ asidi etil ve izopropil esterler, etil laurat, etil miristat, etil palmitat, izopropil laurat, izopropil miristat, izopropil palmitat, tipik olarak kozmetik endüstrisinde kullanılan biyouyumlu organik sıvılar olarak seçilmiştir. $\mathrm{Bu}$ nedenle, bu çalışmanın bir başka amacı, topikal uygulama için en iyi organoleptik ve reolojik özelliklere sahip jeli belirlemektir [2].

\section{Materyal ve Metod}

Tüm kimyasallar ticari kaynaklardan (Merck, Fluka ve Sigma-Aldrich) satın alındı ve aksi belirtilmedikçe daha fazla saflaştırma yapılmadan kullanıld1. Laurik asit etil ester (LEE), laurik asit izopropil ester (LIE), miristik asit etil ester (MEE) ve miristik asit izopropil ester (MIE), palmitik asit etil ester (PEE), palmitik asit izopropil ester (PIE) literatürde açıklanan protokollerle hazırlandı. Arzu edilen yağ asidi izopropil esterleri, katalitik miktarda dehidrate p-toluen sülfonik asit varlığında uygun yağ asidi alkol ile büyük molar fazlalıkta işlenerek yüksek verimle (\% 88-94) elde edildi [3].

Teknikler: (400 MHz) ve 13C (100 MHz) NMR spektrumları BRUKER AV-400 Yüksek Performansl1 Dijital FT-NMR üzerinde kaydedildi ve kimyasal kaymalar ppm olarak rapor edildi. Reolojik ölçümler, bir koni plakalı (20 mm çaplı) Anton PAR MCR 301 gerilme reometresi ile gerçekleştirilmiştir.

\section{Jelatörlerin Sentezi}

\section{Dimetildiglikolat' In Sentezi}

Literatürde tarif edilen sentetik prosedürler, bu esterlerin hazırlanmasında uygulanmıştır [4]. Kuru nitrojen altında $500 \mathrm{~mL}$ metanol ve piridin karışımına, $250 \mathrm{~mL}$ benzen içinde bir diglikolik asit diklorür (17.1 g, $100 \mathrm{mmol}$ ) çözeltisi, 3 saat süre boyunca basınç kontrollü bir damlatma hunisi yoluyla damla damla ilave edildi. Çöken tuz, süzülerek çıkarıldı ve çözücü, döner buharlaştırıcı yardımıyla uzaklaştırıldı. Kalan tortunun özü, soğuk eter $(3$ x $50 \mathrm{~mL})$ ile ekstarsiyon yapılarak organik faza geçirildi. Sonra beyaz bir kat1 ürün elde edildi. Ürünler dietil eter-petrol eterinden (2:1) yeniden kristallendirildi, verim: diglikolat ester için $\% 80$.

\section{N-(1-benzil-2hidroksi-etil)-2[(1-benzil-2hidroksi-} etilkarbomil-metoksi)]-asetamid.

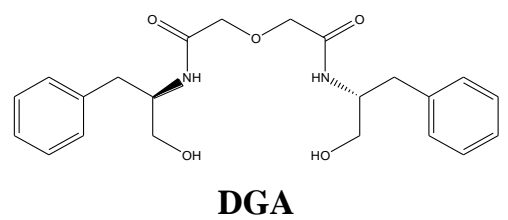

DGA'nın sentezi literatürde bildirilmiştir [5]. Dimetildiglikolat (0.16 g, $1.2 \mathrm{mmol})$, toluen $25 \mathrm{~mL}$ içindeki L-fenilalanilol $(0.71 \mathrm{~g}, 2.5$ mmol) süspansiyonuna eklendi. Deney düzeneğine geri soğutucu ve Dean-Stark aparat1 bağlanarak 2 gün boyunca karıştırıldı. Reaksiyonun ilerleyişi TLC eter / petrol eteri (2: 1) ile izlendi. Çözücü, vakumla buharlaştırılarak uzaklaştırıldı ve kalan tortu, eter ile yıkandı. Ham materyalin saflaştırılması, silikajel üzerinde kolon kromatografisiyle (yıkama sıvis1: eter / petrol eteri 2: 1) gerçekleştirildi. Verim: $72 \%$; erime noktas1: $128-130{ }^{\circ} \mathrm{C} ;[\alpha]^{20}=$ $-39.2(\mathrm{c}=0.03, \mathrm{MeOH}) . \mathrm{C}_{22} \mathrm{H}_{28} \mathrm{~N}_{2} \mathrm{O}_{5}$ için hesaplanan kütle verileri: $\mathrm{C}, 65.98 ; \mathrm{H}, 7.05 ; \mathrm{N}$, 7.00. Bulunan: C, 65.97; H, 7.09; N, 6.97. IR (KBr): v: 3413, 3316, 3243, 3070, 3031, 2931, 
2873, 1643, 1546, 1110, $1045 \mathrm{~cm}^{-1} ; 1 \mathrm{H} \mathrm{NMR}$ $(400 \mathrm{MHz}, \mathrm{CDCl} 3): \delta(\mathrm{ppm})=2.70(2 \mathrm{H}, \mathrm{dd}, J$ $=16, J=8.4 \mathrm{~Hz}), 2.90(2 \mathrm{H}, \mathrm{dd}, J=8.4, J=7.4$ $\mathrm{Hz}), 3.39\{3.46(4 \mathrm{H}, \mathrm{m}), 3.77\{3.89(4 \mathrm{H}, \mathrm{m})$, $3.91\{4.06(2 \mathrm{H}, \mathrm{m}), 4.91$ (br s $2 \mathrm{H}),, 7.15-7.29$ $(10 \mathrm{H}, \mathrm{m}) 7.91(2 \mathrm{H}, \mathrm{d}, J=8.4) ;{ }^{13} \mathrm{C}$ NMR $(100$ $\mathrm{MHz}, \mathrm{CDCl} 3): \delta(\mathrm{ppm})=37.01,52.71,62.89$, 70.84, 126.46, 128.62, 129.54, 139.50, 169.01.

\section{E vitamini ( $\alpha$-tokoferol)}

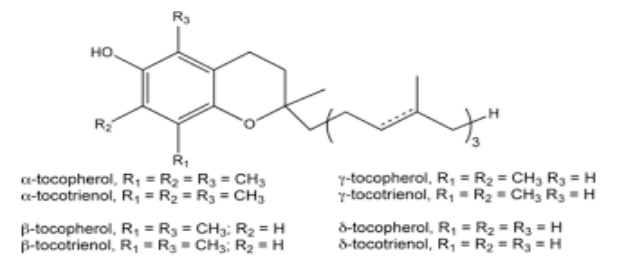

E vitamini ( $\alpha$-tokoferol), yağda çözünür ve ağızdan alındığında membran lipidlerini peroksidasyondan korur. Deride, UV'ye maruz kaldıktan sonra güneş yanığı hücrelerini azalttığı, serbest radikalleri nötralize ettiği ve ayrıca nemlendirici görevi gördüğü gösterilmiştir. Topikal uygulamalar için $\mathrm{C}$ ve $\mathrm{E}$ Vitaminlerinin birleştirilmesinin hem bireysel antioksidanlarını hem de foto koruyucu etkilerini arttırdığına dair önemli kanıtlar vardır [6].

\section{Vitamini}

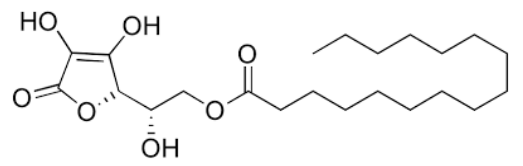

Askorbik asit veya $\mathrm{C}$ vitamini, özellikle sulu ortamda oksidasyonla kolayca parçalanır. $\mathrm{Bu}$ nedenle askorbil palmitat gibi daha kararlı türevlerin kullanılması tercih edilir [7].

\section{Yükleme Deneyleri}

Vitaminlerin jel sistemlerine yüklenmesi literatürde açıklanan prosedürle gerçekleştirildi [8]. $1 \mathrm{~mL}$ jelleşme sivisı içerisine minimum oranda eklenerek jel sisteminin oluşumu sağlanır ve buna mininmum jel konstanrasyonu (MGC) denir. Minimum jel konsantrasyonunda hazırlanan jellere vitaminlerin yüklenmesi ard arda ve yavaşça eklenerek gerçekleştirilir. Daha sonra aşağıdaki denklem kullanılarak yükleme kapasitesi hesaplanır [9].

Yükleme Kapasitesi $=\% \mathrm{w}_{\mathrm{c}} / \mathrm{w}_{\mathrm{g}} \mathrm{x} 100$

wd, jele yüklenen kozmetik maddenin ağırlığıdır ve wg ise jel matrisindeki toplam ağırlıktır.

\section{Reolojik Ölçümler}

jel formülasyonuna vitamin yüklemenin önemli bir yönü olarak, reolojik performanslarındaki bozunma davranışına 1şık tutmak için fiziksel ve yapısal stabiliteyi ölçmektir. Reolojik analiz, farmasötik yarı katıların karakterizasyonunda iyi bilinen bir metodolojidir. Modern reolojik ekipmanlar ve ayrica matematiksel hesaplamalar, reolojiyi, topikal malzeme geliştirmede ve ürün parametrelerini takip etmede güçlü bir araç haline getirmektedir. Kozmesötik formülasyonun amaca uygun olması için minimum düzeyde eksipiyanlar (jelatör, sıvı vb.) kullanılması istenmektedir. DGA jelatörün minimum jel konsantrasyonu göz önünde bulundurularak; sıv1 parafin, MIE ve PEE, kozmesötik yükleme deneylerinde kullanılmış ve kozmesötik taşıyıcı matris olarak değerlendirilmiştir [6]. Bu nedenle LEE ve MIE jellerinde DGA jelleştiricisinin viskoelastik özellikleri gerilim kontrollü reometrede (Anton PAR MCR 301) boşluğun genişliği $0.047 \mathrm{~mm}$ olan ve $20 \mathrm{~mm}$ 'lik bir koni plakada ölçüldü. Numune, reometrenin düz yüzeyine yerleştirildi. Deney, frekans taramasının yanı sira gerinim taramas1 modunda yapıld1. Viskoelastik özelliklerin ölçülmesinden önce, doğrusal viskoelastik bölge (VLR), gerinimin bir fonksiyonu olarak depolama veya elastik modülü ve kayıp veya viskoz modülü ölçülerek belirlendi. Ardından, $G^{\prime}$ ve $G^{\prime}$ ölçümleri, bir frekans tarama testi ile gerçekleştirildi. Frekans tarama testleri, $25^{\circ} \mathrm{C}$ 'de dinamik gerinim tarama ölçümlerinden belirlenen organojel numunelerin doğrusal viskoelastik sisteminde gerçekleştirilmiştir. $\mathrm{Bu}$ frekans tarama testlerinde, elastik modül doğrusal bölge içinde ölçülmüştür, bu nedenle sonuçlar bozulmamış bir jelin özelliklerini açıklamıştır. Salınım deneyler, $1-10 \mathrm{~Hz}$ frekans aralığında, sabit gerilimde $(0,5 \mathrm{~Pa})$, oda sicaklığında (yani $\left.25^{\circ} \mathrm{C}\right)$ ölçülmüştür. Bu salınımlı ölçümler, her bir DGA formülasyonu için elastik modülü $\left(\mathrm{G}^{\prime}\right)$, viskoz modülü $\left(G^{\prime \prime}\right)$ ve karmaşık viskoziteyi $(\eta *)$ elde etmek için kullanılmıştır. LEE ve MIE içinde silindirik şekilli jeller hazırlandı ve 2-3 saat bekletildi. Jel sistemleri için derinlemesine bilgi elde etmek için frekans tarama testinden karmaşık viskozite $\eta *$, karmaşı modül $G *$ ve kayıp faktörü $\left(\operatorname{kayıp} \delta=\mathrm{G}^{\prime \prime} / \mathrm{G}^{\prime}\right)$ yeniden elde edildi. $\mathrm{Bu}$ bağlamda, jelleşme sıvısına bağlı olarak çeşitli organojellerin reolojisi araştırıldı. 
Hazırlanan jellerin elastik modülü $\left(\mathrm{G}^{\prime}\right)$ ve viskoz modülü (G ") Tablo 1'de listelenmiştir. Minimum jel konsantrasyonu $4 \mathrm{mg} / \mathrm{mL}$ 'den daha yüksek konsantrasyonlar sirasiyla MIE ve LEE'deki reolojik ölçümleri keşfetmek için kullanılmıştır. Bunlara ek olarak, salınımlı ölçüm testi, MIE içinde hazırlanmış farklı DGA jelatör konsantrasyonları ile yapılmıştır; benzer şekilde formülasyonların stabilitesini değerlendirmek için frekans süpürme testi DGA / MIE jel sistemi ile yapild1.

Şekil 1. $25^{\circ} \mathrm{C}$ 'de frekansın bir fonksiyonu olarak viamin $\mathrm{C}$ yüklü (1 mgmL-1) ve yüklenmemiş formlarla DGA / MIE jelinin (4 mgmL-1) salınım frekansı ölçümü. Elastisite modülü $\left(\mathrm{G}^{\prime}\right.$, yeşil ve mavi) ve viskozite modülü $\left(G^{\prime}\right.$ ', mor ve kırmızı), sırasıyla $C$ vitamini yüklü ve boş form.

Figure 1. Oscillating frequency measurement of DGA/MIE gel (4 mgmL-1 ) with viamine C loaded (1 mgmL-1) and unloaded forms as a function of frequency at $25 \mathrm{oC}$. Elasticity modulus ( $\mathrm{G}^{\prime}$, green and blue) and viscosity modulus ( $G^{\prime \prime}$, purple and red), vitamine $C$ loaded and unloaded forme respectively.

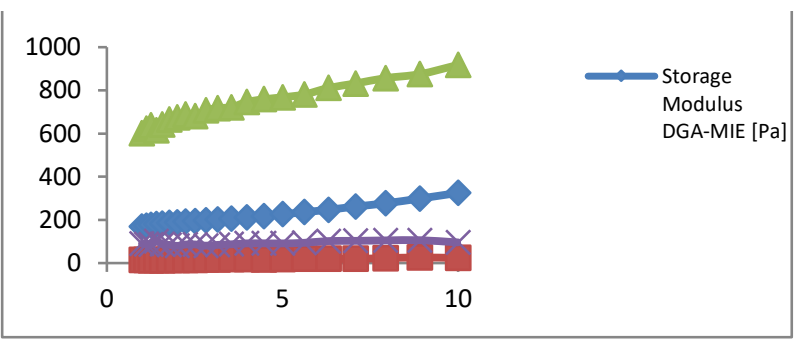

Şekil 2. $25^{\circ} \mathrm{C}$ 'de frekansın bir fonksiyonu olarak viamin E yüklü (1 mgmL-1) ve yüklenmemiş formlarda DGA / MIE jelinin (4 mgmL-1) salınım frekansı ölçümü. Elastisite modülü $\left(\mathrm{G}^{\text {', }}\right.$ yeşil ve mavi) ve viskozite modülü ( $\mathrm{G}^{\prime}$ ', mor ve kırmızı), E vitamini sırasıyla yüklü ve boş form.

Figure 2. Oscillating frequency measurement of DGA/MIE gel (4 $\left.\mathrm{mgmL}^{-1}\right)$ with viamine $\mathrm{E}$ loaded $\left(1 \mathrm{mgmL}^{-1}\right)$ and unloaded forms as a function of frequency at $25{ }^{\circ} \mathrm{C}$. Elasticity modulus ( $\mathrm{G}^{\prime}$, green and blue) and viscosity modulus (G", purple and red), vitamine $\mathrm{E}$ loaded and unloaded forme respectively.

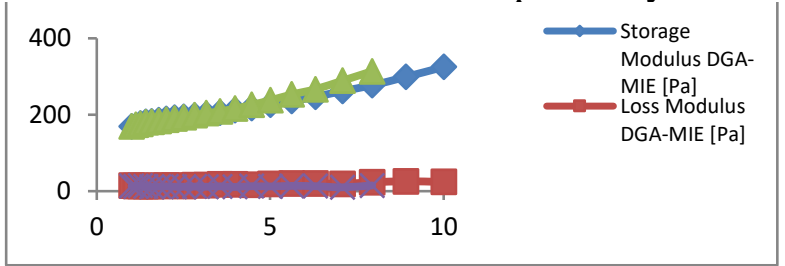

Tablo 1. $\mathrm{G}^{\prime}$ (elastik) ve $\mathrm{G}^{\prime \prime}$ (viskoz) modüllerinin reolojik parametre değerleri, $25^{\circ}$ $C^{\prime}$ de kompleks viskozite $(\eta *)$, Vitamin yüklü ve yüksüz jel formları ile farklı jelatörler / sıv1 kombinasyonu elde edilen reolojik veriler. $(\mathrm{n}=$ $10,2,82 \mathrm{~Hz}$ ).

$\mathrm{Bu}$ sonuçlar, bize bu sistemleri $\mathrm{E}$ vitamini ve $\mathrm{C}$ vitamini (ester türevi, askorbil palmitat) için yeni bir taşıyıcı olarak denemeye teşvik etti. Buna ek olarak, bu organorojelatör, diğer kozmetik ajanların taşıyıcıları olarak hizmet edebilir çünkü karş1lıklı herhangi bir vitamin jelleştirici etkileşimi olmadığ Kozmesötik-taşıyıcı matrisler için en önemli ön koşullardan biridir. Bu sonuçlar, diglikolamidin iyi bir organojelatör olduğunu ve orta enerji ile yeterince kararlı jeller ürettiğini göstermektedir.

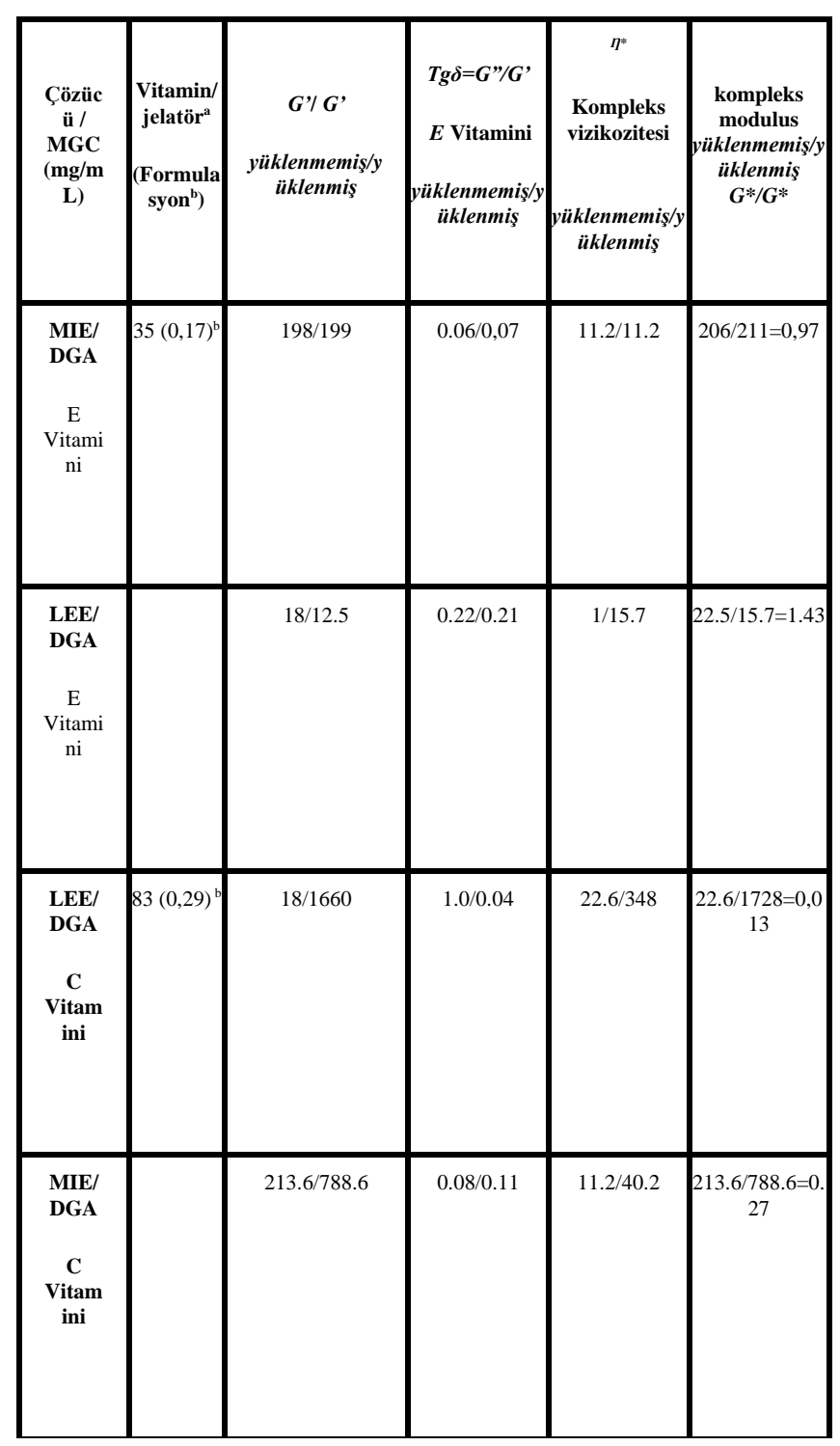




\section{Sonuçlar}

Preparatların

organoleptik

özellikleri, bileşimlerine bağlı olarak değişiklik göstermiştir. $\mathrm{Bu}$ formüller topikal kullanım için tasarlandığından tat belirlenmemiştir. $\mathrm{Bu}$ gözlemler, DGA jellerinin yaşlanmayı geciktiren vitamin $\mathrm{E}$ için uygun taşıyıcı görevi görebileceğini ve vitamin $\mathrm{C}$ (askorbil palmitat) moleküllerinin ester türevinin, her iki sistem için kozmesötik yüklü ve yüklenmemiş jel formlarıyla mükemmel $\operatorname{Tg} \alpha$ değerlerine sahip olduğunu göstermektedir. Ayrıca reolojik verilerin doğru DGA-yağ asidi esteri kombinasyonu seçimi ile ince ayarlanabileceğini göstermiştir. Bu çalışmada, DGA -FAE'leri yaşlanmayı geciktiren malzemeler için taşıyıcı olacak yeni bir platform olarak tanımlama girişiminde bulunulmuştur. Bu amaçla, DGA'nın yeni bir düşük molekül ağırlıklı organojelatör olarak yapılandırılmış doğal malzemelerden hazırlanması ve kozmetik çözücülerde araştırılan jelleșme davranıșları incelenmiștir. Farklı sıvılardaki DGA'nın hazırlanan taşıyıcının reolojik performansının kontrol edilmesini sağladığı gösterilmiştir.

\section{Teşekkür}

Yazar, bu araştırmayı mali olarak desteklediği için Türkiye Bilimsel ve Teknolojik Araştırma Kurumu'na (TÜBİTAK) teşekkür eder (Proje No. 113Z142). Projelerini destekledikleri için Dicle Üniversitesi Araştırma ve Proje Konseyi'ne teşekkürlerini sunar (No. FEN.17.005). Bu araştırmada yapılan reolojik ölçümlerden dolayı Prof. Dr. Adnan Hayaloğlu ve ekibine teşekkür eder. Aynı zamanda reolojik dataların analizinde yardımlarını esirgemeyen Prof. Dr. Halil Hoşgören'e teşekkürlerini sunar.

\section{Kaynaklar}

1. AR Mullaicharam , Geetali Deori , R Uma maheswari, Research Journal of Pharmaceutical, Biological and Chemical Sciences (RJPBCS). 2013,4, (1)1092-1101. ISSN: 0975-8585

2. Welin-Berger, Katayoun,The effect of rheological behaviour of a topical anaesthetic formulation on the release and permeation rates of the active compound, European journal of pharmaceutical sciences. 2001,13,(3), 309-318. DOI:10.1016/S0928-0987(01)00118-X
3. Sanna, V.; Mariani, A.; Caria, G.; Sechi, M. Synthesis and Evaluation of Different Fatty Acid Esters Formulated into Precirol ${ }^{\circledR}$ ATO-Based Lipid Nanoparticles as Vehicles for Topical Delivery. Chem. Pharm. Bull. 2009, 57 (7), 680-684.

4. Mehmet Colak, Deniz BARIŞ, Necmettin PRINCÇÇİĞLU, Halil HOŞGÖREN Novel Barış, D.; Şeker, S.; Hoşsgoren, H.; Toğrul, M. Tetrahedron-Asymmetry. 2010, 21, 1893-1899.

5. bis(aminoalcohol)oxalamide organogelators and their diglycolylamide analogs: evaluation of gelation efficiency in various organic fluids. Turk J Chem. 2017, 41: 658-671 doi:10.3906/kim-1701-76

6. Pinnell SR. Pinnell SR. Cutaneous photodamage, oxidative stress, and topical antioxidant protection. J. Am. Acad Dermatol. 2003; 48:1-19.

7. Gallarate, M.; Carlotti, M.E.; Trotta, M.; Bovo, $\mathrm{S}$. On the stability of ascorbic acid in emulsified systems for topical and cosmetic use. Int. J. Pharm. 1999, 188, 233-241.

8. Serhat Uzan, Deniz Barıs, Mehmet Çolak, Haluk Aydın, Halil Hoşgören. Organogels as novel carriers for dermal and topical drug delivery vehicles. Tetrahedron. 2016, 72, 75177525 .

9. Suzuki, M., Saito, H., and Hanabusa, K. Two component organogelators based on two Lamino acids: effect of combination of L-lysine with various L-amino acids on organogelation behavior. Langmuir. 2009, 25, 8579-8585. doi:10.1021/la8040924. 